\title{
A Low-Noise 492 GHz SIS WAVEGUIDE RECEIVER
}

\author{
C.K. Walker ${ }^{1,3}$, J.W. Kooi ${ }^{1}$, M. Chan ${ }^{1}$, H.G. LeDuc ${ }^{2}$, \\ P.L. Schaffer ${ }^{1}$, J.E. Carlstrom ${ }^{1}$, and T.G. Phillips ${ }^{1}$ \\ 1- Caltech Submillimeter Observatory \\ Division of Physics, Mathematics and Astronomy \\ California Institute of Technology, Pasadena, California 91125
}

2- Center for Space Micorelectronics Technology, Jet Propulsion Laboratory

3- Presently at the University of Arizona, Tuscon AZ 


\title{
A Low-Noise 492 GHz SIS Waveguide Receiver
}

\author{
C. K. Walker ${ }^{1,+}$, J. W. Kooi ${ }^{1}$, M. Chan ${ }^{1}$, H.G. LeDuc ${ }^{2}$, P.L. Schaffer ${ }^{1}$, \\ J.E. Carlstrom ${ }^{1}$, and T.G. Phillips ${ }^{1}$ \\ ${ }^{1}$ California Institute of Technology, Pasadena CA \\ 2 Jet Propulsion Laboratory, Pasadena CA \\ $\uparrow$ Presently at the University of Arizona, Tucson AZ
}

\begin{abstract}
In this paper we discuss the design and performance of an SIS waveguide receiver which provides low noise performance from 375 to $510 \mathrm{GHz}$. At its design frequency of $492 \mathrm{GHz}$ the receiver has a double sideband noise temperature of $\sim 172$ $\mathrm{K}$. By using embedded magnetic field concentrators, we are able to effectively suppress Josephson pair tunneling. Techniques for improving receiver performance are discussed.
\end{abstract}

\section{Introduction}

Over the last decade SIS receivers have been replacing Schottky diode based systems at millimeter and submillimeter wavelengths. SIS junctions have a lower shot noise and a more pronounced D.C. nonlinearity than Schottky diodes, with the result being that mixers constructed with them are more sensitive and require less local oscillator power than their Schottky diode counterparts.

SIS mixers can be constructed using waveguide or an open structure geometry. To date, at all frequencies where they have been built, SIS waveguide mixers provide superior performance. The main advantage waveguide mixers have over open structure designs is that adjustable backshorts can be readily incorporated into the mixer block. Backshorts are usually needed to match the complex impedance, although, recently, structures employing lithographically produced matching networks for waveguide mounted junctions have proved highly successful (Kerr et al. 1987). Open structure mixers typically utilize a combination of lenses and planar antenna structures to couple the incoming radiation to the junction. With this combination of components it is not easy to incorporate an adjustable backshort. Fixed tuned reactive stubs can be fabricated along with the SIS device to tune out the junction's capacitance. A significant advantage of waveguide designs is that well characterized, efficient feedhorns can be used to couple waveguide modes to free space modes. 
Until recently SIS waveguide receivers have been constructed with center frequencies only as high as $345 \mathrm{GHz}$. In this paper we discuss the construction and performance of an SIS waveguide receiver with a center frequency of $492 \mathrm{GHz}$. It is now permanently installed on the Caltech Submillimeter Observatory and has been used for astronomical obscrvations since September 1991.

\section{Receiver Construction}

\section{Optics}

A block diagram of the $500 \mathrm{GHz}$ receiver is shown in Figure 1. The receiver's optics are designed to provide $\mathrm{a}-10 \mathrm{db}$ taper on the edge of the telescope's secondary mirror. The beam from the secondary is reflected from an offset parabola, a flat, and a final offset parabola before entering the cryostat. At the entrance to the cryostat a thin $(\sim 0.2 \mathrm{mil})$ mylar beamsplitter is mounted at $45^{\circ}$ to the signal and local oscillator beams. With this thickness of mylar only $0.5 \%$ of the signal and local oscillator beam is reflected. Therefore only a small fraction of the signal is lost, while almost all the local oscillator power is terminated in an absorbing load.

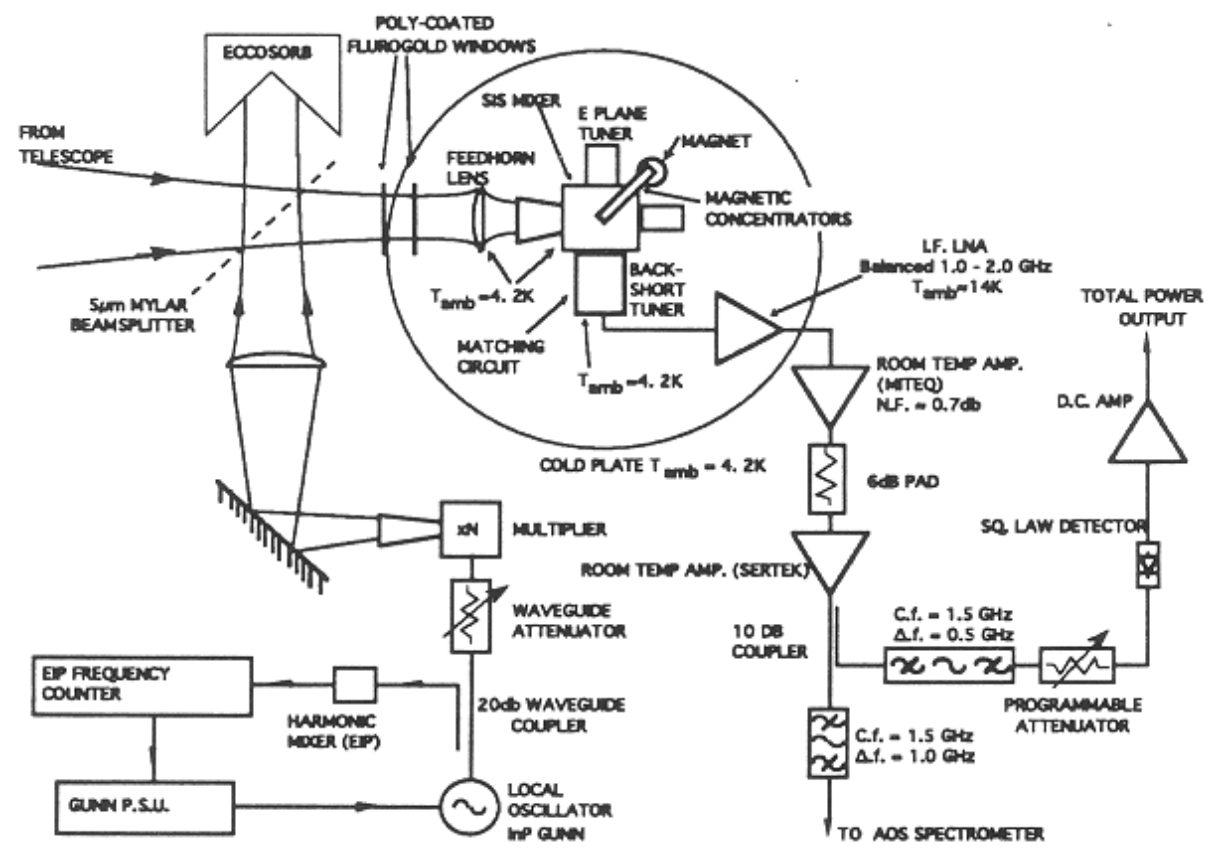

Figure 1

The cryostat vacuum window is a 1.0 mil mylar sheet. The windows on the 12 $\mathrm{K}$ and $77 \mathrm{~K}$ shields are made from disks of fluorogold laminated to a quarter wavelength thickness of black polyethylene. The polyethylene reduces reflection losses. The overall thickness of the fluorogold and black polyethylene disks is 17 mils. The disks serve as near infrared blocking filters for the system. 
The last optical component before the mixer block is a small, low density polyethylene lens mounted in front of the feedhorn. The lens is designed using the equations of Silver (1966) and is located so that it provides frequency independent illumination of the secondary (Goldsmith 1982).

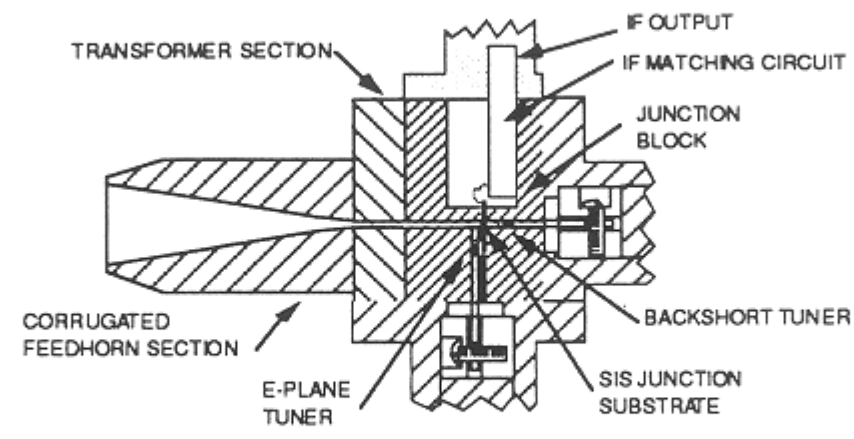

Diagram of Mixer Block

Figure 2

\section{Mixer Block}

A schematic representation of the mixer block is shown in Figure 2. An exploded view of the completed block is shown in Figure 3. The basic design of the waveguide portions of the block follows that of Ellison et al. (1987). The block is divided into five sections along the longitudinal axis of the block. The first section consists of a corrugated feedhorn (Thomas 1978) which terminates in circular waveguide. The beamwidth of the horn, at its $-10 \mathrm{db}$ points is $13.4^{\circ}$. In the second section, the signal passes through a three step, circular to full-height rectangular, quarterwave transformer. This section also contains the waveguide for the E-plane tuner. The center of the E-plane tuner is located $\sim \lambda \mathrm{g} / 2$ in front of the SIS junction. The third section contains the SIS device, the IF impedance matching circuit, magnetic field concentrators, and the waveguide for the backshort. A face-on view of this section is shown in Figure 4. The location of the main circuit elements are indicated. The SIS junction substrate resides in a long 4.5 by 4 mil channel milled across the center of the waveguide. When the junction substrate is placed in the channel, it is oriented so that the junction itself is in the center of the waveguide facing the oncoming radiation. An RF choke is fabricated on the substrate at the same time the junction is made. The choke, shown in Figure 5, is made from a series of high and low impedance sections of microstrip line. Each section is $\lambda / 4$ in length at the RF center frequency. The ground side of the junction is held in place with silver paint. The "hot" side of the junction is connected to the IF matching circuit via a short 1.0 mil gold wire. The wire is silver painted to the last section of the RF choke located on the junction substrate. To keep the silver paint from inadvertently shorting the hot side of the junction substrate to the block, the block is designed so that the last 8 mils of the junction substrate is suspended in free space. 


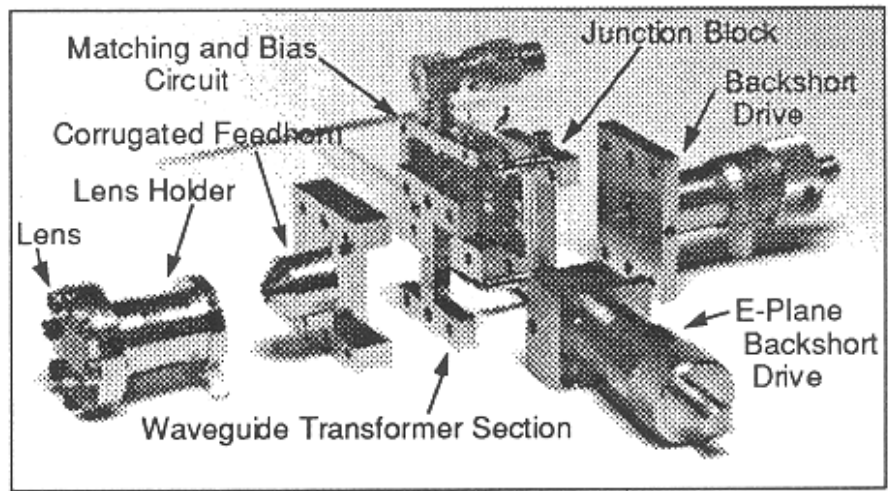

Exploded View of the 490 Block

Figure 3

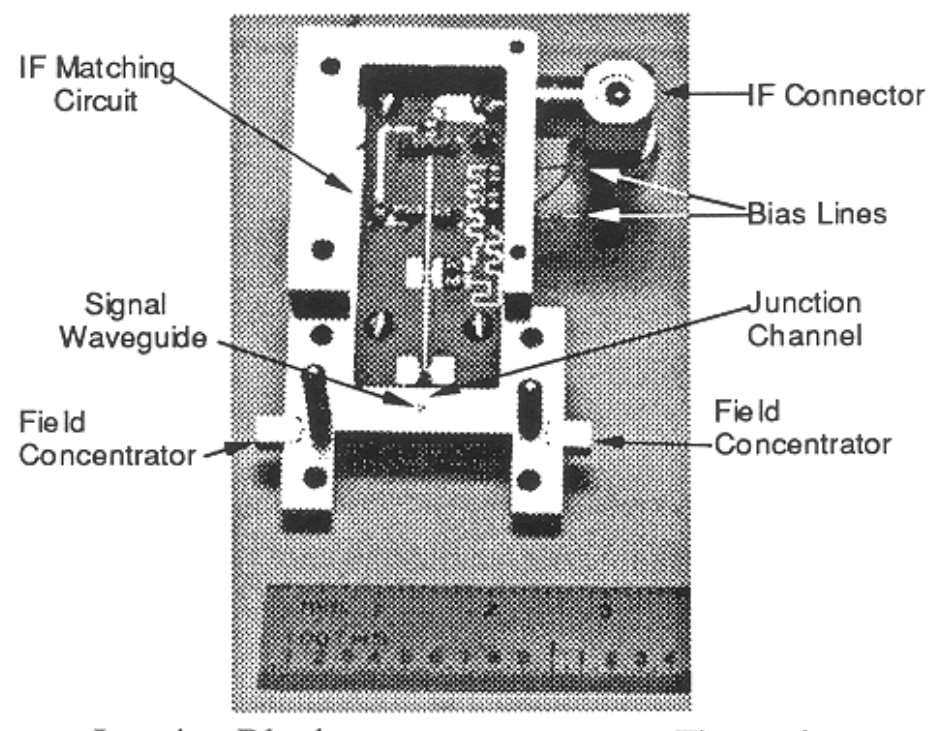

Junction Block

Figure 4

A detailed discussion of the integrated IF matching circuit has been given by Kooi et al. (1992). It utilizes a 5-pole Chebyshev low pass filter and transformer to match the IF impedance of the SIS junction to the input impedance of the IF amplifier $(\sim 50 \Omega)$ over a 1 to $2 \mathrm{GHz}$ frequency range. The IF impedance of the junction is roughly 2.5 times the junction's normal state resistance. The matching circuit is designed for an SIS IF impedance of $\sim 160 \Omega$.

The magnetic field concentrators and the core of the external electromagnet to which they are connected are made out of Cryoperm, a material which retains a high magnetic permeability even at $4 \mathrm{~K}$. To reduce heat loading on the $4 \mathrm{~K}$ cold plate, the magnet's coil is made from niobium wire. The backshorts are non-contacting and were designed using the techniques discussed by Brewer and Rasianen (1982). Non- 
contacting backshorts were chosen over contacting backshorts because of their durability.

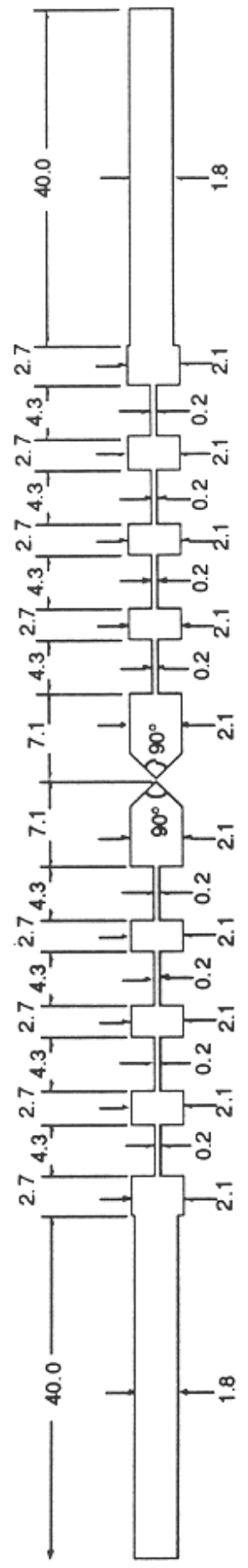

Note: Al dimensions are in mis

$492 \mathrm{GHz}$ Mask Dimensions

Figure 5

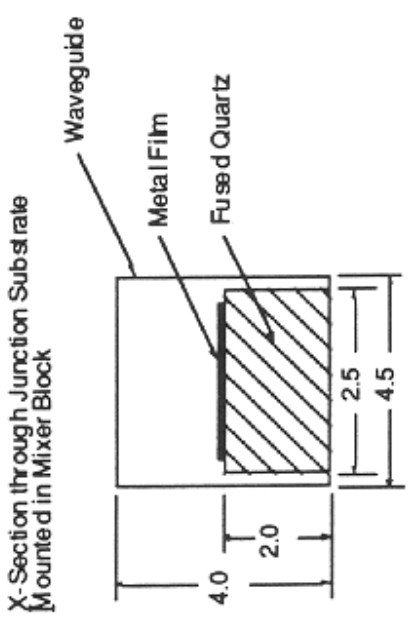




\section{Junction Fabrication}

The $\mathrm{Nb} / \mathrm{ALO}_{\mathrm{x}} / \mathrm{Nb}$ tunnel junctions used in this receiver are planar, submicron area devices defined by electron beam lithography. The junctions are fabricated using a variant of the self-aligned-lift-off trilayer process (Shoji et al. 1983) with modifications for electron beam lithographic patterning of junction area. The $\mathrm{Nb} / \mathrm{ALO}_{\mathrm{x}} / \mathrm{Nb}$ trilayer is deposited in a high vacuum sputter deposition system (base pressure $1.3 \times 10^{-7} \mathrm{Torr}$ ). The $\mathrm{Nb} / \mathrm{Al}$ layers are $\mathrm{dc} / \mathrm{rf}$-magnetron sputtered from 75 $\mathrm{mm}$ diameter targets. The $\mathrm{AlO}_{\mathrm{x}}$ tunnel barrier on the trilayer is formed by an in situ oxidation in an oxygen/argon mixture. A gold layer $(\approx 30 \mathrm{~nm})$ is deposited on the trilayer to act as a contact layer. The junctions are patterned by forming submicron holes in PMMA by electron beam lithography (JEOL JBX5) followed by the deposition and lift-off of chromium metal. The chromium pattern is transferred to an underlying polyamide film using oxygen reactive ion etching (RIE). Junctions are formed by $\mathrm{RIE}$ using a gas mixture containing $\mathrm{CCl}_{2} \mathrm{~F}_{2}$ (chosen for its highly anisotropic etch characteristics) and electrically isolated using thermally evaporated silicon monoxide. Contact wiring is deposited and patterned using RIE to complete the device. Tunnel junctions with areas of $0.25 \mu \mathrm{m}^{2}$ and $0.13 \mu \mathrm{m}^{2}$ were fabricated on on the same wafer

\section{Receiver Performance}

Figure $6 \mathrm{a}$ is a plot of the current versus voltage (I-V) curve of the SIS junction used in the receiver. The solid line is the I-V curve with no local oscillator power applied. The curve has a sharp knee at the voltage $(\sim 2.78 \mathrm{mV})$ corresponding to the band-gap energy of the niobium junction. At the knee, the leakage current through the junction is about $5 \mu \mathrm{A}$. The normal state resistance of the junction is about $90 \Omega$. The dotted line is the I-V curve with local oscillator applied. With local oscillator power applied a single wide photon step is observed below the knee of the curve. The width of this step corresponds to the voltage $(h v / e)$ of a $492 \mathrm{GHz}$ photon $(\sim 2 \mathrm{mV})$. Figure $6 \mathrm{~b}$ is a plot of IF power versus SIS bias voltage. The top curve is the IF power obtained with the receiver looking into a room temperature load $\left(\mathrm{T}_{\mathrm{H}} \sim 280 \mathrm{~K}\right)$. The lower curve is the IF power obtained with the receiver looking into a cold load $\left(\mathrm{T}_{\mathrm{C}} \sim\right.$ $80 \mathrm{~K}$ ). As expected, the IF conversion peak occurs at a bias voltage corresponding to the middle of the first photon step below the gap voltage. The ratio of the IF power obtained with the receiver looking into the room temperature load to the IF power obtained with it looking into the cold load is a measure of the receiver's sensitivity and is often referred to as the $\mathrm{Y}$-factor. The receiver noise temperature is derived from the $\mathrm{Y}$-factor using the following relation.

$$
T_{R}=\frac{T_{H}-Y T_{C}}{Y-1}
$$

At $492 \mathrm{GHz}$ the highest $\mathrm{Y}$-factor obtained with this receiver was 1.84 , which corresponds to a double sideband receiver noise temperature of $\sim 172 \mathrm{~K}$. This value is 
a true receiver noise temperature, no corrections have been made for signal losses in the beamsplitter or input optics, losses resulting from impedance mismatches, or from IF amplifier noise. The bias voltage and current used during this measurement were $2.3 \mathrm{mV}$ and $12 \mu \mathrm{A}$. At this bias voltage the receiver noise temperature increased when the LO power was reduced to a level where the junction current became less than $\sim 9 \mu \mathrm{A}$. Similarly, if the LO power was increased such that the junction current reached a value greater than $16 \mu \mathrm{m}$, the receiver sensitivity decreased.
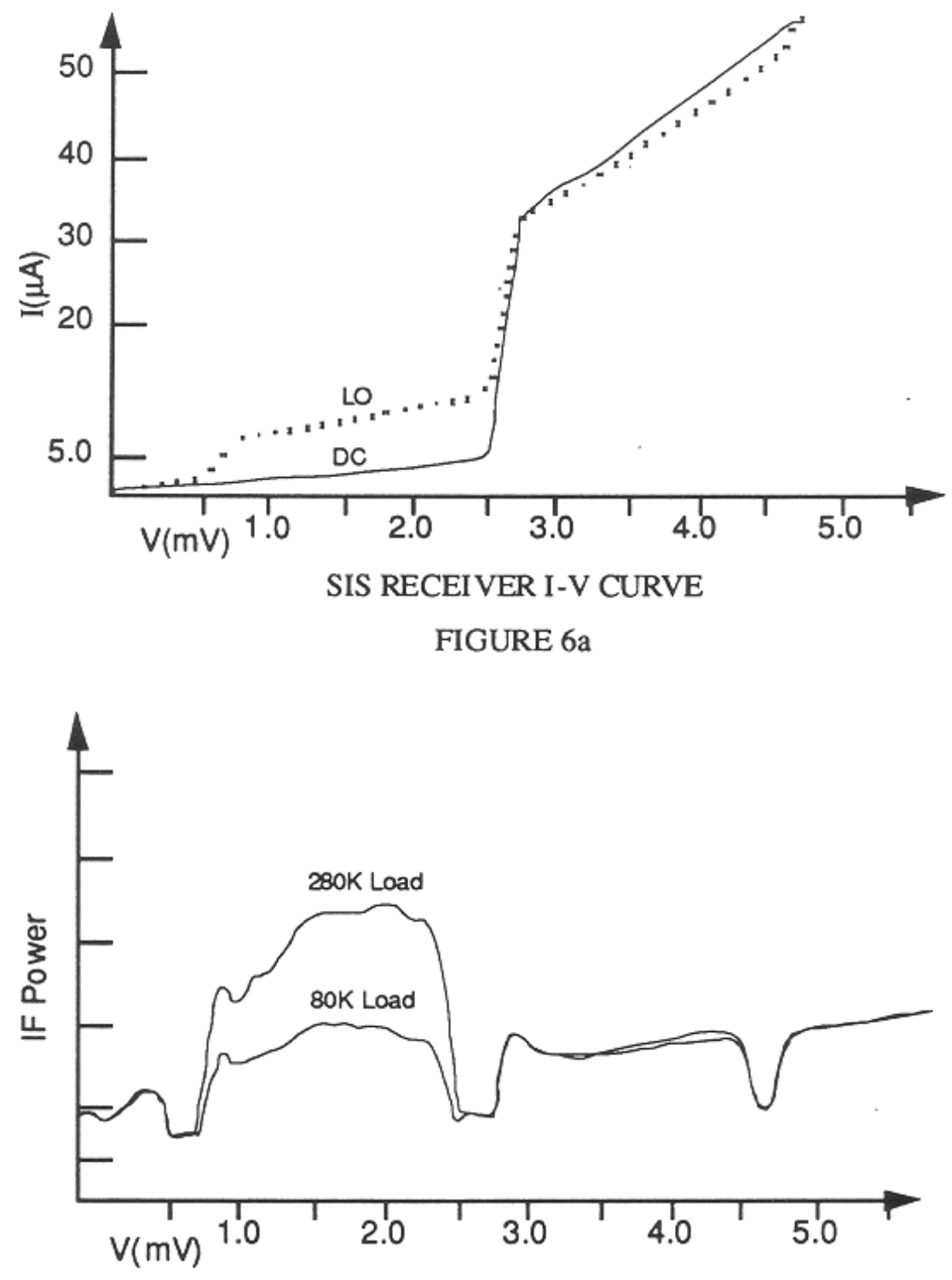

SIS RECEIVER IF POWER vs. BIAS VOLTAGE

FIGURE $6 b$ 
During these measurements the magnetic field strength was adjusted so as to minimize the manifestation on the I-V characteristics of the Josephson pair tunneling current. As the magnetic field strength was increased, the Josephson super-current went through several minima. A minimum in the super-current occurs when one or more magnetic flux quanta are present across the junction. Since more than one minimum was observed, we conclude the magnetic circuit used in this design is capable of placing several flux quanta across the junction. Without the magnetic field the smooth IF power curves of Figure $6 \mathrm{~b}$ become jagged. In Figure 7 we present IF power curves made at $420 \mathrm{GHz}$. Figure 7a shows the smooth IF power curves that can be obtained with an optimized magnetic field. In Figure $7 \mathrm{~b}$ the same IF power curves are plotted, but with the applied magnetic field less than optimum. In 7a and $7 \mathrm{~b}$ we also plot the corresponding junction I-V curve. The small dips in the IF power curve of Figure 7a become more prominent in Figure 7b, and occur at voltages where Josephson steps are seen in the pumped I-V curve. The association between the dips and the Josephson steps indicates that these structures are the result of Josephson effect tunneling. These results show that, even at high frequencies, a magnetic field can be used to effectively suppress Josephson pair currents in small area junctions.

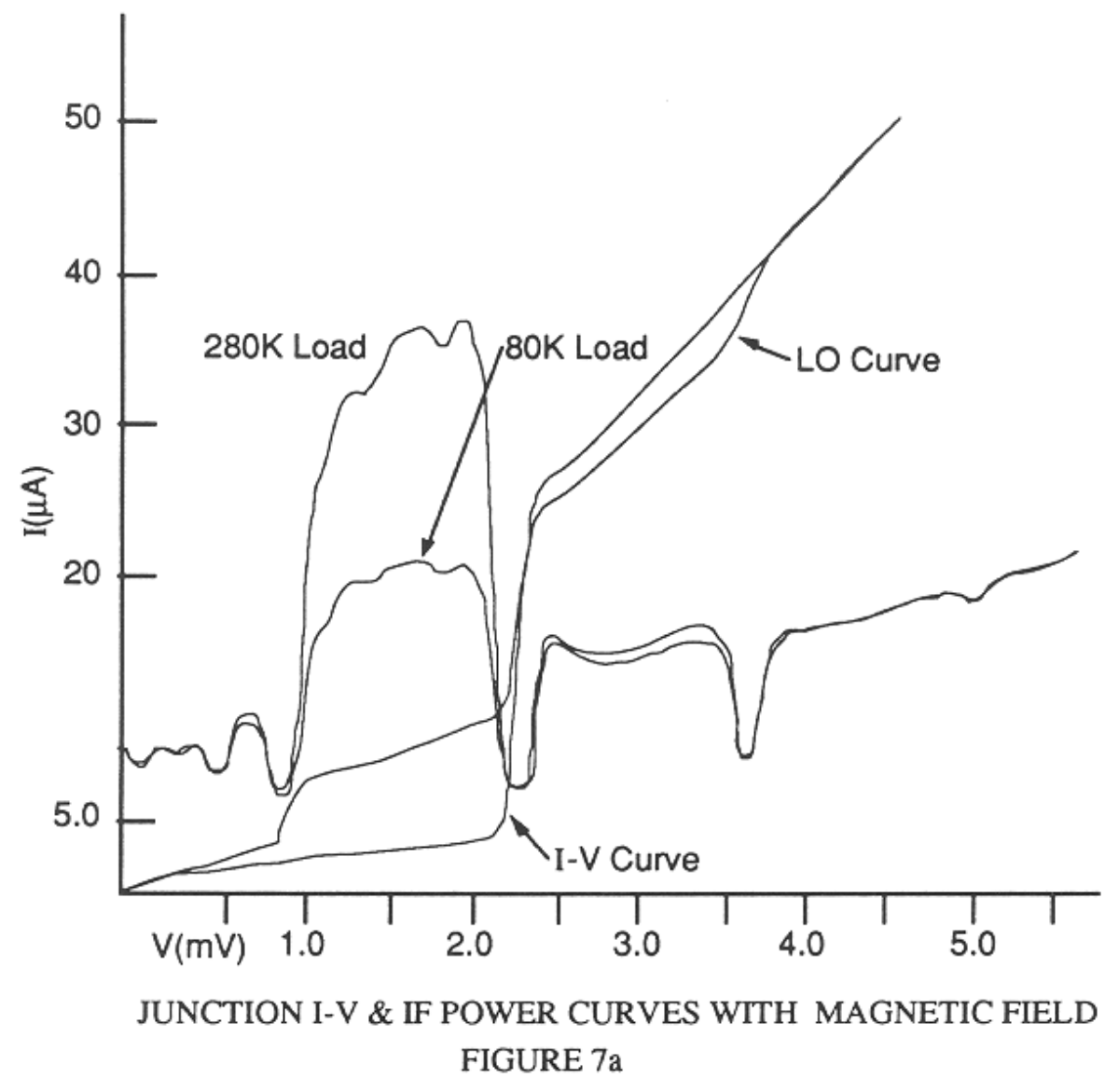




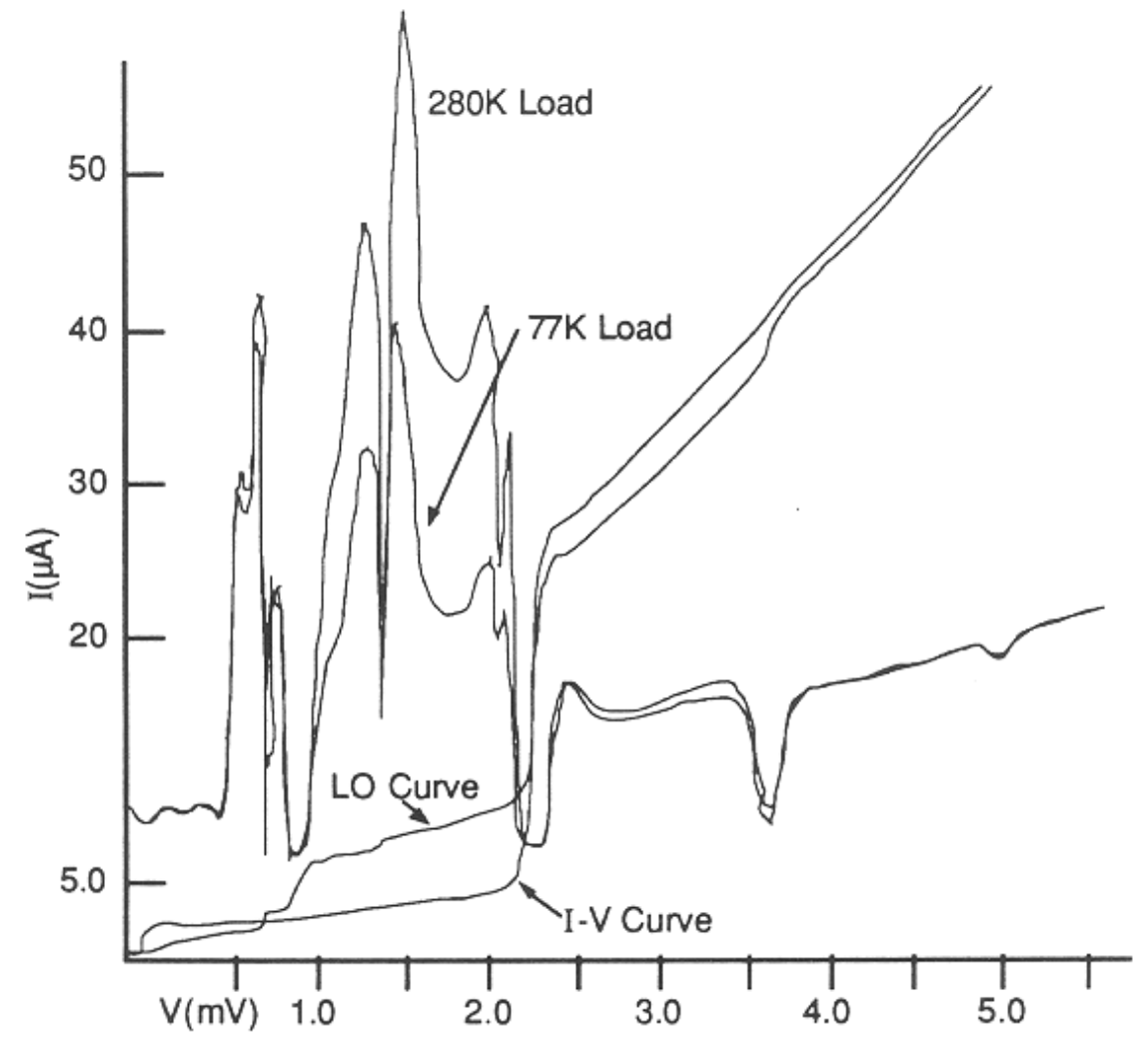

JUNCTION I-V \& IF POWER CURVES WITH REDUCED MAGNETICFIELD

FIGURE 7b

In Figure 8 we show spectrum analyzer measurements of the receiver bandpass from $0.9 \mathrm{GHz}$ to $2.1 \mathrm{GHz}$. The lower curve in the figure is the bandpass with the receiver looking into a room temperature $(280 \mathrm{~K})$ load. The bandpass is fairly flat, with a IF power variation of only $\sim 3.5 \mathrm{db}$ from 1 to $2 \mathrm{GHz}$. The upper curve is a plot of the receiver's Y-factor across the IF band. This curve is essentially flat, indicating the receiver's sensitivity is constant across the band.

In Figure 9 we present a double sideband, $500 \mathrm{MHz}$ wide spectrum taken with the receiver on the Caltech Submillimeter Observatory toward the young stellar source Orion IRC2. The center frequency of the spectrum is $492.16 \mathrm{GHz}$. An acousto-optical spectrometer was used to produce the spectrum. An atomic line and several molecular lines were observed. They are identified in Figure 9. The ordinate is in units of antenna temperature $(\mathrm{K})$ and the abscissa is in units of frequency $(\mathrm{GHz})$. The total on source integration time was $\sim 1.3$ minutes. At this frequency, the system noise is dominated by the atmosphere. During the time this spectrum was taken the single sideband noise temperature on the sky, which includes the noise contributions of the atmosphere, telescope, and receiver, was $\sim 5000 \mathrm{~K}$. 


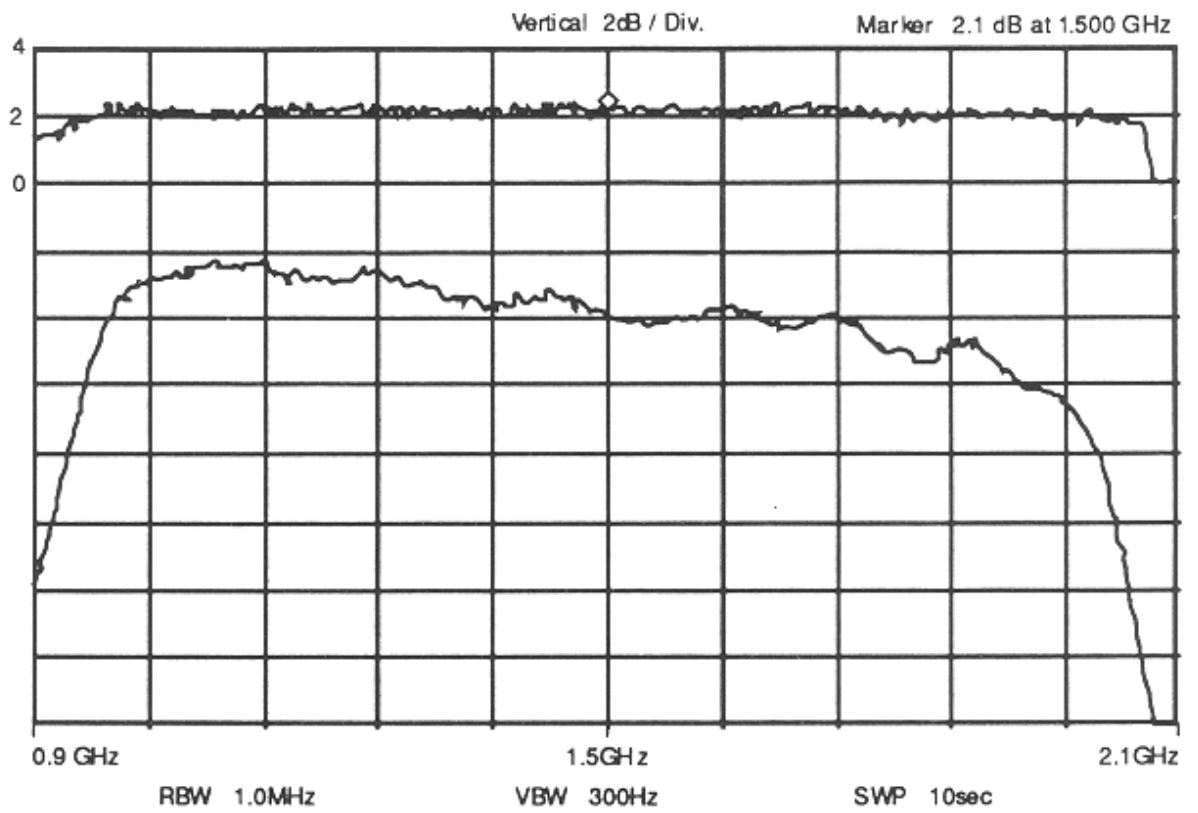

Receiver Bandpass Plot

Figure 8

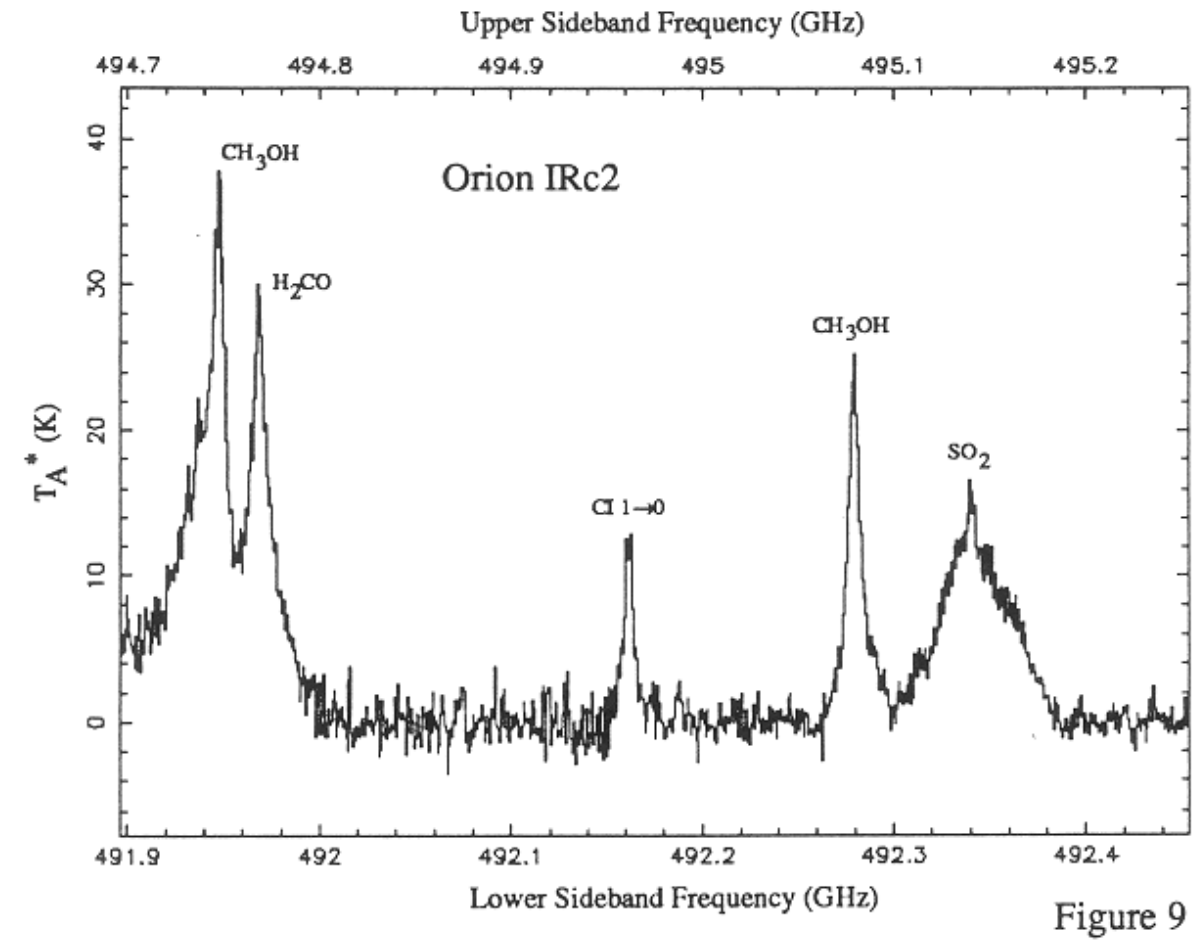


The receiver noise temperature, $T_{R}$, is determined by a number of factors. These include the mixer noise temperature, $\left(\mathrm{T}_{\mathrm{M}}\right)$, the conversion loss in the mixer $\mathrm{C}_{\text {Loss }}$ (Conv. Loss), the noise temperature of the first IF amplifier $\left(\mathrm{T}_{\mathrm{IF}}\right)$, and the coupling efficiency between the IF port of the junction and the input port of the first IF amplifier ( $\left.\eta_{\text {IF }}\right)$.

\section{Receiver Performance Comparison}

\begin{tabular}{lccc} 
Parameter & $230 \mathrm{Nb}$ & $345 \mathrm{~Pb}$ & $492 \mathrm{Nb}$ \\
\hline $\mathrm{R}_{\mathrm{n}}(\Omega)$ & 82 & 54 & 85 \\
$\mathrm{~T}_{\mathrm{R}}(\mathrm{K})$ & 48 & 159 & 176 \\
$\mathrm{~T}_{\text {mix }}(\mathrm{K})$ & 34 & 129 & 123 \\
$\mathrm{C}_{\text {Loss }}(\mathrm{db})$ & 3.1 & 8.3 & 8.9 \\
$\mathrm{~T}_{\text {mix }}$ Cor. $(\mathrm{K})$ & 26 & 91 & 95 \\
$\mathrm{~T}_{\text {IF }}(\mathrm{K})$ & 7.0 & 4.2 & 6.8 \\
IF Contrib. $(\mathrm{K})^{14}$ & 14 & 30 & 53
\end{tabular}

Table 1

In Table1 we list the values of these parameters for the receiver described in this paper and for the two other SIS waveguide receivers presently in use at the Caltech Submillimeter Observatory. The values of $\mathrm{T}_{\mathrm{M}}, \mathrm{T}_{\mathrm{IF}}, \mathrm{C}_{\mathrm{Loss}}, \eta_{\mathrm{IF}}$ and were calculated using the shot noise technique described by Tucker and Feldman (1985). The other two receivers in the table were designed with center frequencies of 230 and $345 \mathrm{GHz}$. All three receivers use the same basic double stub tuner design. Like the $492 \mathrm{GHz}$ receiver, the $230 \mathrm{GHz}$ system employs a niobium junction and the IF matching circuit described by Kooi et al. (1992). The $345 \mathrm{GHz}$ receiver uses a lead based SIS junction fabricated by Ron Miller at AT\&T Bell Laboratories. This receiver uses an older, less efficient IF matching circuit. In the table $R_{n}$ refers to the normal state resistance of the SIS junction used in each receiver. $T_{M}$ Cor. refers to the mixer temperature corrected for the different beam splitter thicknesses used in each receiver. The value of $T_{R}$ for the $230 \mathrm{GHz}$ system is a factor of 3 to 4 less than the $T_{R}$ achieved with the $492 \mathrm{GHz}$ system. The decrease in system performance at 492 $\mathrm{GHz}$ is due to a factor of $\sim 3$ increase in $\mathrm{C}_{\mathrm{Loss}}$ and $\mathrm{T}_{\mathrm{M}}$. The performance of the 345 and $492 \mathrm{GHz}$ receivers are comparable. This similarity in performance is most likely due to the niobium junction in the $492 \mathrm{GHz}$ receiver having a better high frequency response than the lead junction used in the $345 \mathrm{GHz}$ receiver. 


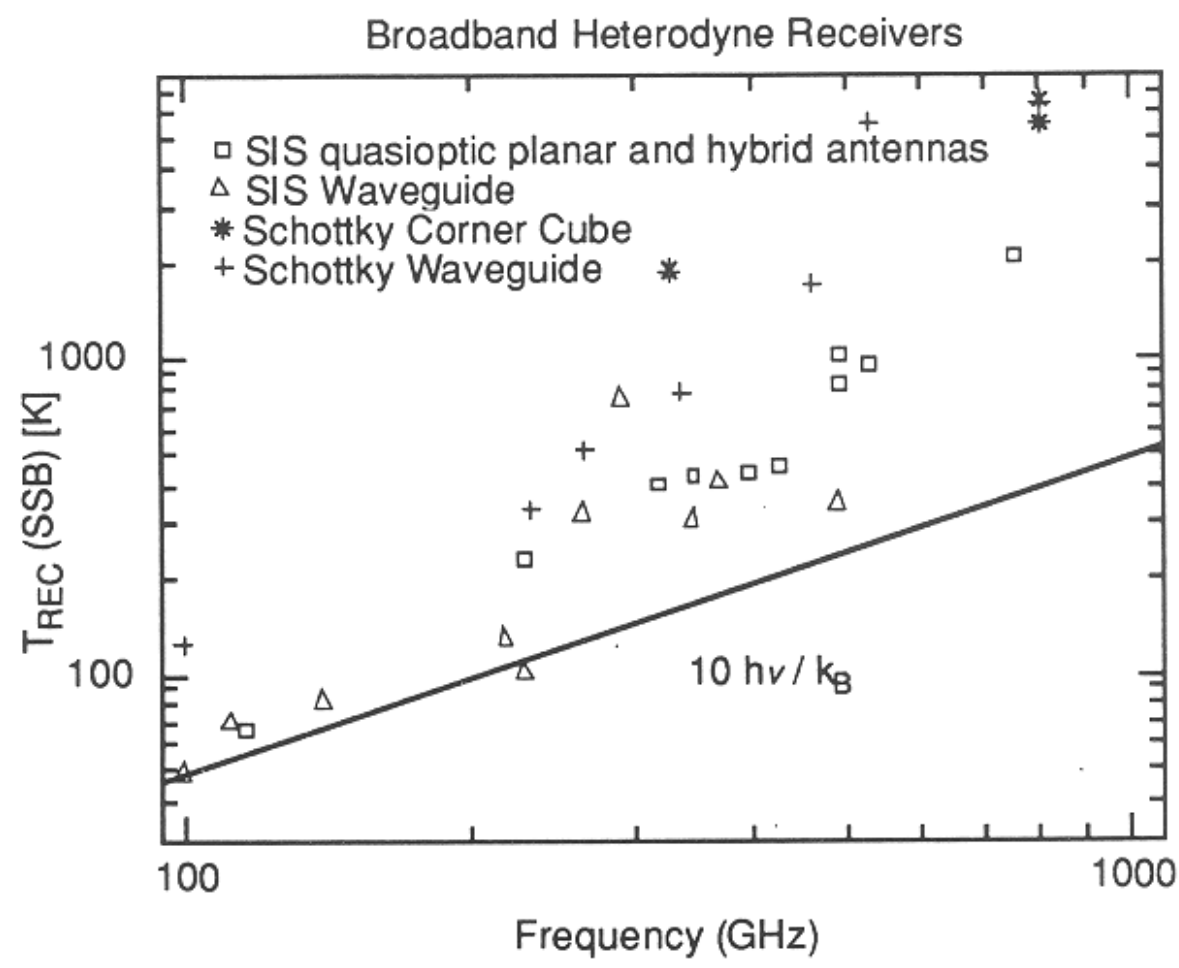

In Figure 10 we compare the performance of the $492 \mathrm{GHz}$ receiver to other receivers operating at millimeter and submillimeter wavelengths. At each frequency where they have been built, SIS waveguide receivers have proven to be the most sensitive. These receivers typically operate with noise temperatures 10 to 14 times the quantum noise limit $(h v / e)$. The receiver reported in this paper continues this trend. We have also tested the $492 \mathrm{GHz}$ receiver at $376 \mathrm{GHz}$ and $420 \mathrm{GHz}$. We obtained $T_{R}$ values of 238 and $212 \mathrm{~K}$ respectively. (These measurements were taken at sea level where the physical temperature of the mixer was $\sim 0.62 \mathrm{~K}$ higher than at the altitude where the $172 \mathrm{~K}, 492 \mathrm{GHz}$ noise temperature measurements were made.) These noise temperatures include all the losses in the system. If we simply compensate for the difference in the thickness of beamsplitter used in the measurements, then we infer a noise temperature of $200 \mathrm{~K}$ at $376 \mathrm{GHz}$ and $168 \mathrm{~K}$ at $420 \mathrm{GHz}$. These results suggest the receiver will have its optimum performance between 420 and $492 \mathrm{GHz}$.

\section{Improving Receiver Performance}

Lower values of $T_{R}$ would be achieved if we could reduce $T_{I F}$, increase $\eta_{I F}$, or decrease either $C_{\text {Loss }}$ and/or $T_{M}$. In a properly designed amplifier, the value of $T_{I F}$ is a function of the quality of the HEMT devices used and the desired IF bandwidth. In the receiver described here the value of $\eta_{\mathrm{IF}}$ is almost unity, so there is not much to be gained in improving the design of the IF matching circuit. Significantly lower noise temperatures could be achieved if the conversion loss of the mixer were reduced. 
Lower conversion losses can be obtained by improving the impedance match between the junction and waveguide embedding impedance. In the current design this match is achieved by adjusting the positions of the two backshorts. The ultimate quality of the impedance match depends on the performance of the backshorts. Since this receiver will be tuned several times a day, non-contacting backshorts were used to reduce wear. The backshorts are insulated from the block by a thin $(\sim 16 \mu \mathrm{m})$ layer of mylar tape. The performance of the backshorts could be improved (perhaps at the sacrifice of durability) by using a thinner insulating material. Ellison et al. (1991) have recently demonstrated that a 1 to $2 \mu \mathrm{m}$ layer of silicon dioxide evaporated onto metallic backshorts serves as an effective insulating layer and improves backshort performance. An improvement in the $\mathrm{rf}$ match could also be achieved by reducing the capacitance of the SIS junction. The most direct way of doing this is by reducing the size of the junction. If the normal state resistance of the junction is to remain the same, the thickness of the insulating layer must be decreased simultaneously. These last two statements are equivalent to saying we need a high current density junction with a low $\omega R \mathrm{RC}$. The limiting factor in obtaining small, high current density junctions is the junction fabrication process itself. However, over the past few years great strides have been made in improving processing techniques. There is every indication that this trend will continue. The effective capacitance that the backshorts need to tune out can also be reduced by fabricating broadband, inductive stubs on the SIS junction itself. This technique has worked well at frequencies below $300 \mathrm{GHz}$ (Kerr et al. (1987)). However, as one goes up in frequency, the dimensional tolerances on these matching circuits becomes more severe, making fabrication difficult. Even so, recent work by Büttgenbach et al. (1992) suggests that if a broadband rf matching network is included, this technique can be used effectively even at submillimeter wavelengths. Indeed, we plan to test a mixer block like the one discussed here with a broadband, two section, RF transformer matched SIS junction in the near future. Another way to reduce the effective capacitance is to use a series array of junctions. Two difficulties with this technique are that the array elements must be similar (a fabrication challenge) and the required LO power increases as the square of the number of array elements. At submillimeter wavelengths, where it can be difficult to generate LO power, the extra power needed to drive a series array can become prohibitive.

If there were no Josephson pair tunneling, one might expect that an SIS bias voltage corresponding to the center of the first photon step below the gap would provide the best receiver noise temperatures. However, we find that even with an optimized magnetic field, the best receiver noise temperatures are found at bias voltages close to the gap voltage. To some degree this result is due to the matching requirements of the waveguide and IF matching network. However, this dependence of the receiver noise temperature on bias voltage suggests that, while significantly suppressed by the magnetic field, Josephson pair tunneling does add noise to the system. To avoid this problem, SIS junctions with larger bandgap energies are needed. 


\section{Summary}

We have constructed an SIS waveguide receiver which provides low noise performance from $375 \mathrm{GHz}$ to $510 \mathrm{GHz}$. The receiver is a facility instrument at the Caltech Submillimeter Observatory, where it has been in use since September 1991. The SIS junction used in the receiver is a $0.25 \mu \mathrm{m}^{2}$ niobium trilayer device with a current density of $\sim 10^{4} \mathrm{~A} \mathrm{~cm}^{-2}$. At its design frequency of $492 \mathrm{GHz}$, the receiver has a double sideband noise temperature of $\sim 172 \mathrm{~K}$. By embedding magnetic field concentrators in the mixer block, we are able to put several quanta of magnetic flux across the SIS junction. By adjusting the strength of the magnetic field we are able to effectively suppress Josephson pair tunneling. The success of this receiver suggests that SIS waveguide receivers can provide low noise performance at even shorter wavelengths.

This work was supported by NSF contract AST 9015755 to the CSO and a gift from AT\&T to purchase the spectrum analyzer used to perform some of the measurements.

\section{References}

Brewer, M. K. and Raisanen, A. V. 1982, IEEE. Trans. Microwave Theory and Techniques, 30, 708.

Büttgenbach, T. H., LeDuc, H.G., Maker, P.D., and Phillips, T.G. 1992, IEEE Appl. Superconductivity, submitted.

Ellison, B. N., Litle, L. T., Mann, C. M., and Matheson, D. N. 1991, Electronics Letters, 27, 139.

Ellison, B. N. and Miller, R. E. 1987, Int. J. Infrared and Millimeter Waves, 6, 697.

Goldsmith, P.F. 1982, in, Infrared and Millimeter Waves

Kerr, A. R., Pan, S. K., and Feldman M. J. 1987, Int. J. Infrared and Millimeter Waves, 9, 203.

Kooi, J. W., Chan, M., Phillips, T. G., Bumble, B., and LeDuc, H. G. 1992, preprint.

Shoji, A., Kosaka, F., Shinoki, M., Aoyagi, M. and Hayakawa, H. 1983, IEEE Trans. Magnetism, 19, 827.

Silver, S. 1966, IEE Electromagnetic Waves Series, 19, 95.

Thomas, B. M. 1978, IEEE Trans. Antennas and Propagation, 26, 367. 